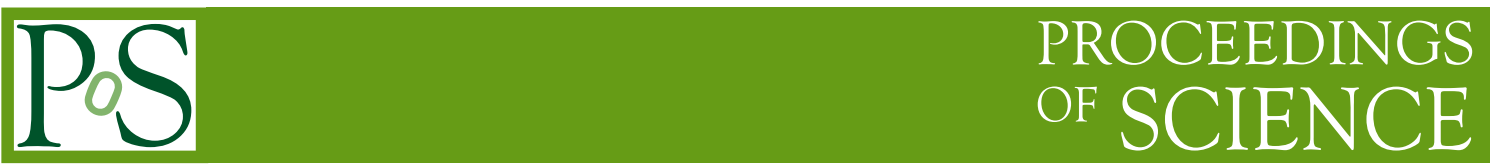

\title{
Dynamics of Kaluza-Klein Towers in the Early Universe
}

\section{Keith R. Dienes}

Department of Physics, University of Arizona, Tucson, AZ 85721 USA

Department of Physics, University of Maryland, College Park, MD 20742 USA

E-mail: dienes@email.arizona.edu

\section{Jeff Kost*}

Center for Theoretical Physics of the Universe, Institute for Basic Science, Daejeon 34126 Korea

E-mail: jeffkostaibs.re.kr

\section{Brooks Thomas}

Department of Physics, Lafayette College, Easton, PA 18042 USA

E-mail: thomasbdelafayette.edu

In this talk, we report on the results of a study [1,2] of the early-universe cosmology of a KaluzaKlein (KK) tower of scalar fields in the presence of a mass-generating phase transition, focusing on the time-development of the total tower energy density (or relic abundance) as well as its distribution across the different KK modes. We find that both of these features are extremely sensitive to the details of the phase transition and can behave in a variety of ways significant for late-time cosmology. In particular, we find that the interplay between the temporal properties of the phase transition and the mixing it generates are responsible for both enhancements and suppressions in the late-time abundances of KK modes, sometimes by many orders of magnitude. ${ }^{\dagger}$

The 39th International Conference on High Energy Physics (ICHEP2018)

4-11 July, 2018

Seoul, Korea

\footnotetext{
* Speaker.

${ }^{\dagger}$ This research was supported in part through DOE Grant DE-FG02-13ER41976, NSF Grant PHY-1720430, and the NSF IR/D program. Opinions and conclusions expressed herein do not reflect any funding agencies.
} 


\section{Introduction}

The presence of additional light scalar degrees of freedom is a common feature of many extensions of the Standard Model (SM). These scalars are often light due to symmetries of the highscale theory which prevent them from acquiring masses. As a result, masses for these particles must be generated by dynamical processes at lower scales which break these symmetries - often in conjunction with a cosmological phase transition. This generically leads to a non-trivial timedependence for the masses of these fields, the details of which can have a significant impact on their late-time energy densities. While the detailed dynamics of mass generation can have important consequences even in scenarios involving only a single light scalar, the situation becomes far richer in scenarios involving many fields, such as the Kaluza-Klein (KK) towers of states in theories with extra dimensions. Namely, the dynamics of mass generation mixes these states in a timedependent way, leading to a continual redistribution of energy density among the scalars during mass-generation, which can have profound effects on the late-time abundance of these fields [1]. In this talk, we study the early-universe cosmology of a KK tower of scalar fields undergoing a mass-generating phase transition, focusing on the time-development of the total tower energy density as well as its distribution across the different KK modes. Further details beyond the results presented here can be found in Refs. [1,2].

\section{The Framework}

The framework in which our analysis will take place is similar to that considered in Ref. [4], and is essentially a KK-oriented extension of the framework considered in Ref. [1]. Specifically, we consider a flat, five-dimensional spacetime geometry of the form $\mathscr{M} \times S^{1} / \mathbb{Z}_{2}$, where $\mathscr{M}$ denotes our usual four-dimensional Minkowski spacetime and where $S^{1} / \mathbb{Z}_{2}$ denotes an orbifolded circle of length $2 \pi R$. We shall also assume that the Standard-Model (SM) fields are brane-localized at the orbifold fixed point at $x^{5}=0$. Within this geometry we consider a five-dimensional scalar field $\Phi\left(x^{\mu}, x^{5}\right)$. While it is natural to assume a five-dimensional shift symmetry $\Phi \rightarrow \Phi+c$ for constant $c$, interactions between $\Phi$ and SM fields can lead to an effective four-dimensional mass for $\Phi$ on the brane. These are typically generated as the result of some brane-localized dynamics which explicitly breaks the symmetry, so that the effective five-dimensional Lagrangian contains a term

$$
\mathscr{L}_{\text {eff }}(\Phi) \supset-\delta\left(x^{5}\right) \cdot \mathscr{V} \cdot \frac{1}{2} m^{2}(t)|\Phi|^{2},
$$

where $\mathscr{V} \equiv 2 \pi R$ is the compactification volume. The behavior of $m(t)$ is ultimately determined by the details of the non-perturbative dynamics on the brane and thus highly model-dependent.

Assuming that $\Phi$ has even parity under the orbifold action $x^{5} \rightarrow-x^{5}$, we perform a KK reduction into a tower four-dimensional fields $\left\{\phi_{k}\right\}$ which have the time-dependent mass matrix $\mathscr{M}_{k \ell}^{2}=k \ell \delta_{k \ell} M_{c}^{2}+r_{k} r_{\ell} m^{2}(t)$, with $M_{c} \equiv 1 / R$ and $r_{k} \equiv \sqrt{2}^{1-\delta_{0 k}}$. It follows that in a flat FriedmanRobertson-Walker (FRW) spacetime the zero-momentum modes obey

$$
\ddot{\phi}_{k}+3 H(t) \dot{\phi}_{k}+\sum_{\ell=0}^{\infty} \mathscr{M}_{k \ell}^{2}(t) \phi_{\ell}=0
$$



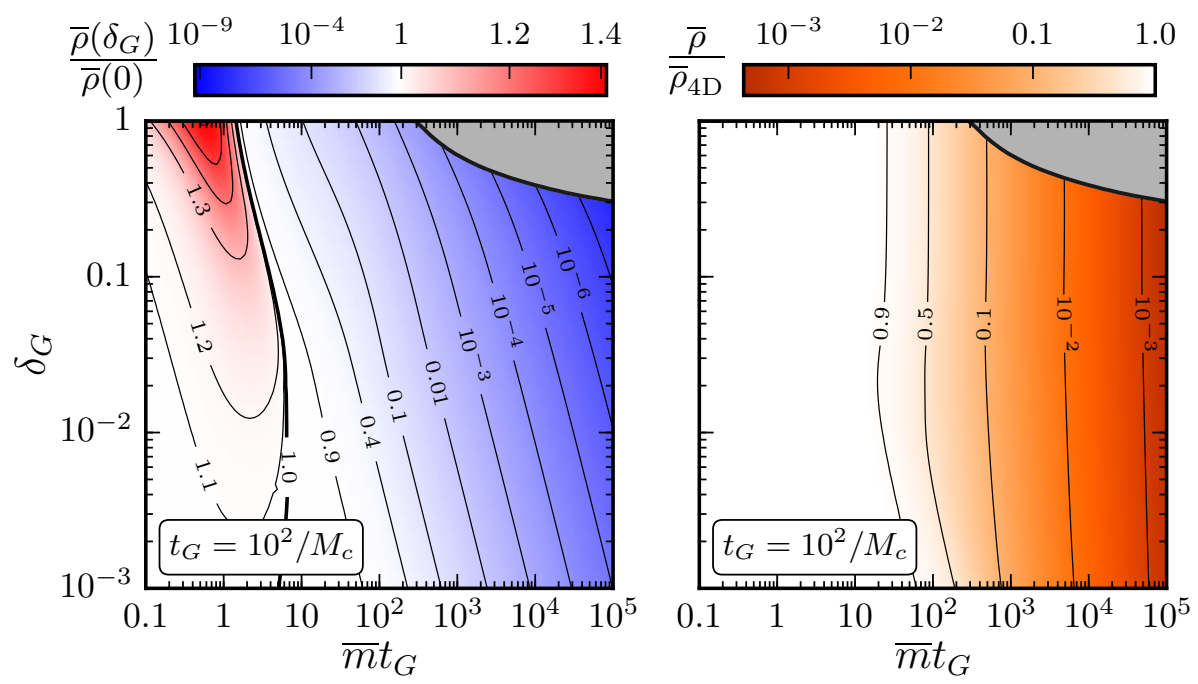

Figure 1: The late-time energy density $\bar{\rho}$ of the full KK tower, plotted in the $\left(\bar{m}_{G}, \delta_{G}\right)$ plane for $t_{G}=10^{2} / M_{c}$. The left and right panels show contours of $\bar{\rho} / \bar{\rho}\left(\delta_{G}=0\right)$ and $\bar{\rho} / \bar{\rho}_{4 \mathrm{D}}$, respectively.

where $H(t) \approx \kappa / t$ is the Hubble parameter with a constant $\kappa$ determined by the cosmological epoch. The time-dependence of $\mathscr{M}(t)$ induces an unavoidable mixing between the different modes, since even the mass-eigenstate basis is continually changing. While in principle any initial conditions are possible, the existence of the shift symmetry $\Phi \rightarrow \Phi+c$ prior to mass generation suggests a fixed non-zero vacuum expectation value (VEV) $\langle\Phi\rangle \neq 0$, which corresponds to only a single non-zero initial condition $\left\langle\phi_{0}\right\rangle \equiv \sqrt{2 \pi R}\langle\Phi\rangle$. Furthermore, we shall assume $m(t)$ is generated at some time $t_{G}$ and unfolds over a duration $\Delta_{G}$. Our eventual results will be largely insensitive to the particular form of $m(t)$, but for concreteness we choose [1]

$$
m(t)=\frac{1}{2} \bar{m}\left\{1+\operatorname{erf}\left[\frac{1}{\sqrt{2} \delta_{G}} \log \left(\frac{t}{t_{G}}\right)\right]\right\}
$$

where $\delta_{G} \equiv \Delta_{G} /\left(\sqrt{2 \pi} t_{G}\right)$ and $\bar{m}$ is the mass at asymptotically late times.

\section{Summary of Results}

We now briefly summarize some of our results. Although the numerical solutions for Eq. (2.2) with a time-dependent mass matrix can only be found for a finite number of modes $N$, we may recover results for the full KK tower by numerically reconstructing the asymptotic $N \rightarrow \infty$ limit.

We shall present our results in several different ways. First, in Fig. 1, we show the total latetime energy density $\bar{\rho}$ as a fraction of $\bar{\rho}\left(\delta_{G}=0\right)$ (left panel) or $\bar{\rho}_{4 \mathrm{D}}$ (right panel), the numerical result for the energy density in the absence of the extra dimension. Our first observation is that while a quantity such as $\bar{\rho}\left(\delta_{G}=0\right)$ might indeed be a useful approximation for $\bar{\rho}$ in certain regions of parameter space, such an approximation can fail badly in others. For example, we see that the abrupt $\left(\delta_{G}=0\right)$ approximation works well for small $\bar{m} t_{G} \lesssim 0.1$ even if $\delta_{G}$ is sizable but otherwise either significantly underestimates or overestimates the true late-time energy density $\bar{\rho}$, the latter 

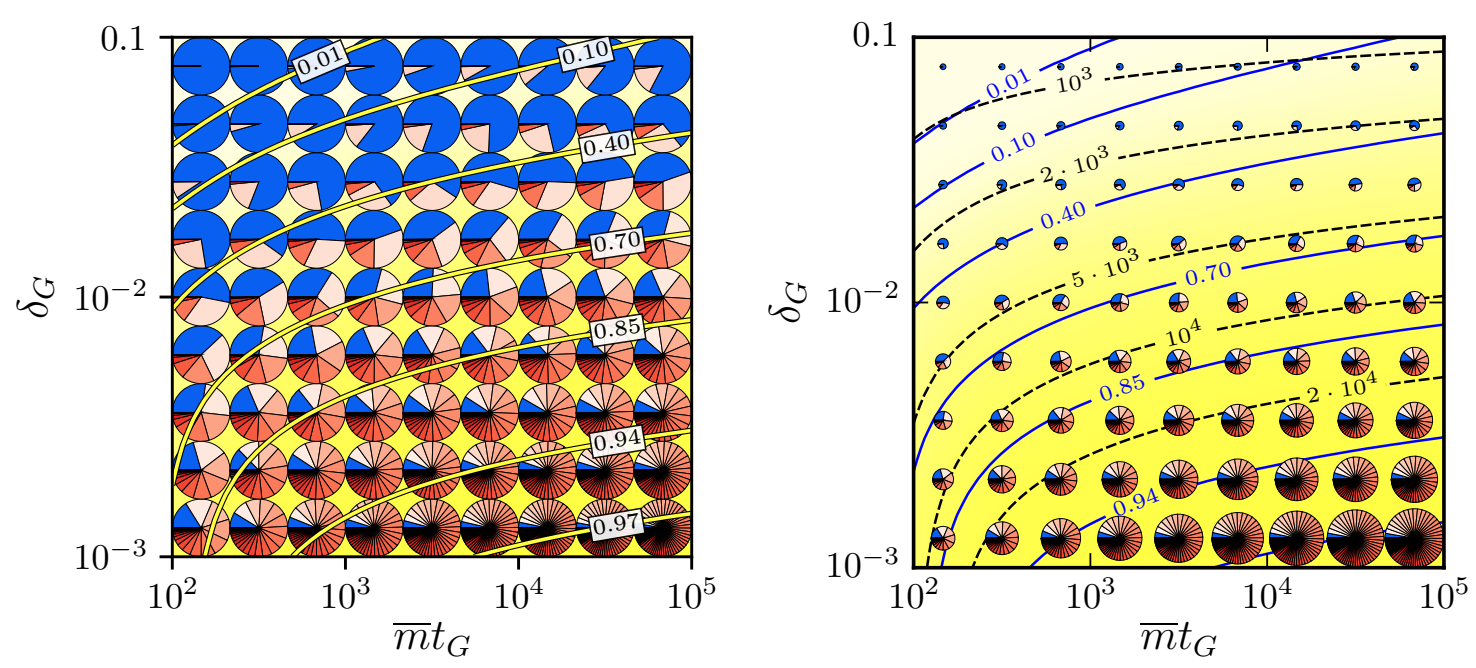

Figure 2: Left panel: A representation of the distribution of energy densities $\bar{\rho}_{\lambda}$ across the KK tower, calculated for a "lattice" in the $\left(\bar{m} t_{G}, \delta_{G}\right)$ plane. Each pie indicates how $\bar{\rho}$ is distributed across the modes, with blue slices indicating the lightest mode and darker-red slices indicating heavier modes. The yellow contours give values of $\bar{\eta} \equiv 1-\max _{\lambda} \bar{\rho}_{\lambda} / \bar{\rho}$. Right panel: Similar plot, but with the pie charts rescaled in proportion to $\bar{\rho}$. Contours of $\bar{\rho}$ (dashed black lines) as well as $\bar{\eta}$ (solid blue lines) are included.

often by many orders of magnitude. Similar observations can be made for $\rho_{4 \mathrm{D}}$, which shows that the effect of the extra dimension is to suppress the total energy density.

We are also interested in the distribution of this total energy density across the modes of our KK tower. We define the "tower fraction" $\eta \equiv 1-\max _{\lambda} \rho_{\lambda} / \rho$, the fraction of the total abundance which is carried by all but the most abundant mode in the tower. The late-time values of these tower fractions for the full KK tower are plotted in the contours of Fig. 2 for $t_{G}=10^{2} / M_{c}$. As a result, it is the region with large $\bar{m} / M_{c}$ and relatively small $\delta_{G}$ for which the total energy density ends up distributed most broadly across the different states in the KK tower at late times. In Fig. 2 the distribution of $\bar{\rho}$ across the different modes is also illustrated through the set of pie charts corresponding to different locations throughout parameter space. We see from this figure that the energy density is preferentially captured by the lightest mode as $\delta_{G}$ increases or $\bar{m} t_{G}$ decreases, but more democratically distributed across the modes of the tower otherwise. We see, then, that a wide variety of energy-density distributions across the KK tower are possible and can be realized simply by adjusting the parameters associated with the mass-generating phase transition.

\section{References}

[1] K. R. Dienes, J. Kost and B. Thomas, Phys. Rev. D 93, 043540 (2016) [arXiv:1509.00470]; K. R. Dienes, J. Kost and B. Thomas, AIP Conf. Proc. 1743, 020003 (2016).

[2] K. R. Dienes, J. Kost and B. Thomas, Phys. Rev. D 95, 123539 (2017) [arXiv:1612.08950 [hep-ph]].

[3] K. R. Dienes, E. Dudas and T. Gherghetta, Phys. Rev. D 62, 105023 (2000) [hep-ph/9912455].

[4] K. R. Dienes and B. Thomas, Phys. Rev. D 85, 083523 (2012) [arXiv:1106.4546 [hep-ph]]; K. R. Dienes and B. Thomas, Phys. Rev. D 85, 083524 (2012) [arXiv:1107.0721 [hep-ph]]. 